\title{
Four-Factor Prothrombin Complex Concentrate Reduces Time to Procedure in Vitamin K Antagonist-Treated Patients Experiencing Gastrointestinal Bleeding: A Post Hoc Analysis of Two Randomized Controlled Trials
}

\author{
Majed A. Refaai, ${ }^{1}$ Truptesh H. Kothari, ${ }^{1}$ Shana Straub, ${ }^{1}$ Jacob Falcon, ${ }^{2}$ \\ Ravi Sarode, ${ }^{3}$ Joshua N. Goldstein, ${ }^{4}$ Andres Brainsky, ${ }^{5}$ Laurel Omert, ${ }^{5}$ \\ Martin L. Lee, ${ }^{6}$ and Truman J. Milling ${ }^{2}$ \\ ${ }^{1}$ University of Rochester Medical Center, Rochester, NY, USA \\ ${ }^{2}$ Seton Dell Medical School Stroke Institute, Dell Children's Medical Center, University Medical Center at Brackenridge, \\ Austin, TX, USA \\ ${ }^{3}$ University of Texas Southwestern Medical Center, Dallas, TX, USA \\ ${ }^{4}$ Massachusetts General Hospital, Boston, MA, USA \\ ${ }^{5}$ CSL Behring, King of Prussia, PA, USA \\ ${ }^{6}$ UCLA Fielding School of Public Health, Los Angeles, CA, USA
}

Correspondence should be addressed to Majed A. Refaai; majed_refaai@urmc.rochester.edu

Received 4 May 2017; Accepted 6 August 2017; Published 19 September 2017

Academic Editor: Marco L. A. Sivilotti

Copyright (C) 2017 Majed A. Refaai et al. This is an open access article distributed under the Creative Commons Attribution License, which permits unrestricted use, distribution, and reproduction in any medium, provided the original work is properly cited.

\begin{abstract}
Introduction. To investigate the impact of a 4 -factor prothrombin complex concentrate (4F-PCC [Beriplex $\left.{ }^{\circledR} / \mathrm{Kcentra}^{\circledR}\right]$ ) versus plasma on "time to procedure" in patients with acute/severe gastrointestinal bleeding requiring rapid vitamin K antagonist (VKA) reversal prior to invasive procedure. Methods. A post hoc analysis of two phase III trials of 4F-PCC versus plasma in patients with acute/severe gastrointestinal bleeding. The treatment arms were compared for study treatment volume, infusion times, and time from start of study treatment to procedure. Results. Analysis included 42 patients (plasma, $n=20 ; 4 \mathrm{~F}-\mathrm{PCC}, n=22$ ). Median (interquartile range) infusion time was significantly shorter for the 4F-PCC group than for the plasma group $(16[13,26]$ min versus $210[149,393] \mathrm{min} ; P<0.0001)$. Median infusion volumes were significantly smaller $(103[80,130] \mathrm{mL}$ versus 870 [748, $1001] \mathrm{mL} ; P<0.0001)$ and median time from study treatment initiation to first procedure was significantly shorter in the 4F-PCC group than in the plasma group $(17.5[12.8,22.8]$ versus $23.9[18.5,62.0] \mathrm{h} ; P=0.037)$. Conclusions. In this analysis of patients with acute/severe gastrointestinal bleeding requiring urgent VKA reversal prior to an invasive procedure, 4F-PCC (compared with plasma) was associated with smaller infusion volumes, shorter infusion times, and reduced time to procedure.
\end{abstract}

\section{Introduction}

Anticoagulants are routinely prescribed for the treatment and prevention of thromboembolic events. However, acute bleeding events in patients treated with oral anticoagulants are common [1]. The reported annual incidence of bleeding in anticoagulated patients is $15-20 \%$ [2]; major bleeding complications occur with an incidence of 1.7-3.4\% [3]. In the United States, bleeding events in patients anticoagulated with vitamin $\mathrm{K}$ antagonists (VKAs) account for more than 60,000 annual emergency room visits [4].

Gastrointestinal (GI) bleeding is the most common major bleeding complication of VKA therapy $[5,6]$; in the recent results published from the Outcomes Registry for Better Informed Treatment of Atrial Fibrillation (ORBIT-AF), GI bleeds represented $38 \%$ of major bleeding events in patients receiving warfarin [7]. GI bleeding is three times more common in patients with an international normalized ratio (INR) 
$>3$ than in those with INR 2-3 [5]. VKA-treated patients who experience acute major bleeding require rapid VKA reversal via the restoration of vitamin $\mathrm{K}$-dependent coagulation factors (VKDFs); this can be achieved by administering plasma or prothrombin complex concentrates (PCCs).

Though widely used, plasma has several disadvantages when used for VKA reversal, including time delays due to blood group typing and thawing of frozen plasma, the need for large volumes and the associated long infusion times to achieve the necessary factor levels, and increased risk of transfusion reactions, such as volume overload and transfusion-related acute lung injury $[8,9]$. PCCs are lyophilized products that are administered in smaller volumes over shorter periods of time; they are either activated or nonactivated. Activated PCCs are indicated for treatment of hemophilia A or B with inhibitors. Nonactivated PCCs are either 3-factor (3F-PCC, containing significant quantities of factors II, IX, and X) or 4-factor (4F-PCC, containing factors II, IX, X, and clinically relevant amounts of factor VII $[9,10])$; these were initially developed for use in people with a congenital deficiency in VKDFs when purified specific coagulation factor is not available [11-15], with some now also being used for prevention or treatment of bleeding associated with VKA treatment $[12,14,15]$.

Two multinational, multicenter phase IIIb clinical trials compared 4F-PCC with plasma for urgent VKA reversal $[16,17]$. 4F-PCC was found to be noninferior to plasma for effective hemostasis and superior to plasma for rapid INR reduction in the study of patients with acute major bleeding [17] and superior to plasma for both of these endpoints in the study of patients needing VKA reversal prior to an urgent surgery or invasive procedure [16]. GI (and other nonvisible) bleeding was the most common type of bleeding reported in the acute major bleeding study, accounting for over $60 \%$ of bleeding events [17]. GI bleeding also occurred in the study of patients needing VKA reversal prior to an urgent surgery or invasive procedure [16]. This post hoc analysis evaluates the subset of patients at two US sites who had GI bleeding in either of the trials.

\section{Methods}

2.1. Study Design. Full details of the design of the acute major bleeding study (NCT00708435) and the urgent surgical or invasive interventions study (NCT00803101) have been published $[16,17]$. Patients of both studies were randomly assigned $(1: 1)$ to receive either 4F-PCC (Kcentra, Beriplex $\mathrm{P} / \mathrm{N}$, CSL Behring, Marburg, Germany) or plasma. Both studies were sponsored by CSL Behring and performed in accordance with local ethics regulations; written informed consent was obtained from or on behalf of all patients.

Using data from two US sites that were major recruiters in the phase IIIb trials (University of Rochester Medical Center [URMC], Rochester, New York, and the Seton Family of Hospitals [SFH], Austin, Texas), we performed a post hoc analysis of patients who had GI bleeding events, to investigate the impact of 4F-PCC versus plasma treatment on time to procedure.
TABLE 1: Dose of study treatment per baseline INR.

\begin{tabular}{lcc}
\hline Baseline INR & $\begin{array}{c}\text { 4F-PCC dose, IU of factor } \\
\text { IX per kg body weight }^{\text {a }}\end{array}$ & $\begin{array}{c}\text { Plasma dose, mL per } \\
\mathrm{kg} \mathrm{body} \mathrm{weight}^{\mathrm{a}}\end{array}$ \\
\hline 2 to $<4$ & 25 & 10 \\
$4-6$ & 35 & 12 \\
$>6$ & 50 & 15 \\
\hline
\end{tabular}

${ }^{\mathrm{a}}$ Dose calculation based on $100 \mathrm{~kg}$ body weight for patients weighing $>100 \mathrm{~kg}$. Maximum dose $\leq 5000 \mathrm{IU}$ of factor IX (4F-PCC) or $\leq 1500 \mathrm{~mL}$ (plasma). $4 \mathrm{~F}-\mathrm{PCC}$, four-factor prothrombin complex concentrate; INR, international normalized ratio.

2.2. Patients. Inclusion criteria for the original studies have been published previously [16, 17]. GI bleeds were experienced by $113 / 212$ (53\%) patients in the acute major bleeding study. As bleeding events were not an inclusion criterion for the surgery study, such events were not systematically reported by all sites in that study. Patients from two study sites (URMC and SFH) who experienced GI bleeding events were included in this post hoc analysis. Together, these sites enrolled 112 patients (30\%) for the phase III studies. Chart review and data abstraction for this post hoc analysis were performed by site personnel without additional funding.

2.3. Treatment. As previously described, on day 1, each patient received the assigned study treatment dosed according to baseline INR and body weight (Table 1) [16, 17]. 4F-PCC was administered as a single intravenous dose, with a maximum infusion rate of $3 \mathrm{IU} / \mathrm{kg}$ per min (equivalent to $8.4 \mathrm{~mL} / \mathrm{min}$ [18]). Plasma was infused intravenously with a study-protocol-recommended rate of $1 \mathrm{U}$ per $30 \mathrm{~min}$ interval in the acute bleeding study [17] and as rapidly as possible and at the discretion of the treating clinical team in the urgent surgical procedure study [16]. All patients were to receive vitamin $\mathrm{K}$ : in the bleeding study, by slow intravenous infusion dosed according to 2008 American College of Chest Physicians guidelines (5-10 mg) [19] or local clinical practice if different; in the surgery study, $2-10 \mathrm{mg}$ (either oral or IV) at the clinician's discretion.

2.4. Assessments. Full details of the assessments performed for this study have been previously described [16, 17]. The primary endpoint of both studies was hemostatic efficacy and the coprimary endpoint was rapid INR reduction $(\leq 1.3$ at $0.5 \mathrm{~h}$ after the end of study treatment infusion). In this post hoc analysis of patients experiencing GI bleeding events requiring surgical or invasive procedures, we assessed dose, volume, duration of infusion, type of procedure, and timings (from admission to initiation of study treatment and to the start of procedure and from initiation of study treatment to start of procedure and total hospital stay duration). Procedures performed in this cohort were endoscopic, including esophagogastroduodenoscopy [EGD], colonoscopy, or sigmoidoscopy; one small bowel follow-through was also performed. We also report safety outcomes for this cohort.

2.5. Statistical Analysis. Between-group differences for study treatment infusion volume, study treatment infusion time, 
time from admission to study treatment, time from admission to first procedure, and time from start of study treatment to first procedure were assessed by the Wilcoxon ranksum test. Between-group differences in hemostatic efficacy and INR reduction were evaluated using the chi-squared test/Fisher's exact test, depending on the cell sizes in the corresponding contingency table. A two-tailed 5\% significance level was used throughout. This post hoc analysis was not powered to support the comparisons performed, so we treated testing of data as descriptive rather than inferential.

Quantitative data were summarized using mean/median and standard deviation/interquartile range (IQR).

\section{Results}

3.1. Patients. The intention-to-treat (ITT) efficacy population of the acute bleeding study and the urgent surgery/procedure study comprised 202 and 168 patients, respectively; the same number of patients $(n=185)$ received 4F-PCC as received plasma $[16,17]$. This post hoc analysis included a total of 42 patients from the two study sites who experienced GI bleeding events; of these, 22 (52\%) patients received 4F-PCC and $20(48 \%)$ received plasma. The 4F-PCC group included $20(90.9 \%)$ patients from the acute major bleeding study and 2 (9.1\%) from the urgent surgery/procedure study. The plasma group included $17(85 \%)$ patients from the acute major bleeding study and $3(15 \%)$ from the urgent surgery/procedure study. Baseline data and characteristics were broadly similar between treatment groups in this analysis (Table 2). Median baseline INR in the acute major bleeding study was 4.6 and 3.2 for the 4F-PCC and plasma groups, respectively, with corresponding values of 2.5 and 2.8 for these groups in the urgent surgery/procedure study.

3.2. Study Treatments. The majority of patients in this analysis received a $4 \mathrm{~F}-\mathrm{PCC}$ dose of $25 \mathrm{IU} / \mathrm{kg}(11 / 22,50 \%)$ or a plasma dose of $10 \mathrm{~mL} / \mathrm{kg}(11 / 20,55 \%)$. The study treatment doses are described fully in Table 3.

As shown in Figure 1(a), the mean study product infusion volume was approximately 8 -fold smaller for patients receiving 4F-PCC than for those receiving plasma $(P<0.0001)$. Median (IQR) infusion volumes were $102.8(80.0,130.0) \mathrm{mL}$ and $870.0(747.5,1000.5) \mathrm{mL}$ for $4 \mathrm{~F}-\mathrm{PCC}$ and plasma, respectively.

The mean study treatment infusion time was approximately 13 -fold shorter for the $4 \mathrm{~F}-\mathrm{PCC}$ group than the plasma group $(P<0.0001$, Figure $1(\mathrm{~b}))$. The median (IQR) infusion times were $16(13,26) \mathrm{min}$ in the 4F-PCC group and $210(149$, 393) min in the plasma group.

3.3. Timing. The mean time between admission and start of study treatment was comparable for patients receiving 4F-PCC and plasma, as shown in Figure 2(a). The median (IQR) time from admission to initiation of study treatment was $4.0(3.1,5.9) \mathrm{h}$ for $4 \mathrm{~F}-\mathrm{PCC}$ and $4.1(3.2,4.8) \mathrm{h}$ for plasma $(P=0.64)$.

The mean time between hospital admission and first procedure was significantly shorter for the 4F-PCC group than the plasma group $(P=0.011)$, as shown in Figure 2(b).
TABLE 2: Patient demographics and baseline disease characteristics.

\begin{tabular}{lcc}
\hline & $\begin{array}{c}\text { Plasma } \\
(n=20)\end{array}$ & $\begin{array}{c}\text { 4F-PCC } \\
(n=22)\end{array}$ \\
\hline Study site, $n(\%)$ & $13(65)$ & $11(50)$ \\
$\quad$ SFH & $7(35)$ & $11(50)$ \\
$\quad$ URMC & $17(85)$ & $20(90.9)$ \\
Study, $n$ (\%) & $3(15)$ & $2(9.1)$ \\
$\quad$ Acute bleeding study & & \\
$\quad$ Urgent surgery/procedure study & $75(11 ; 51,92)$ & $73(14 ; 33,96)$ \\
Age, years & $11(55)$ & $9(41)$ \\
$\quad$ Mean (SD; range) & & \\
Female gender, $n$ (\%) & $29.9(6.5)$ & $30.1(13.5)$ \\
Body mass index, kg/m ${ }^{2}$ & & \\
$\quad$ Mean (SD) & $8.6(2.9)$ & $8.5(2.1)$ \\
Initial (admission) Hgb, g/dL & & \\
$\quad$ Mean (SD) & $26.5(8.1)$ & $25.8(6.2)$ \\
Initial (admission) Hct, \% & & \\
$\quad$ Mean (SD) & $4.7(3)$ & $5.5(3.3)$
\end{tabular}

Time on VKA treatment prior to study entry, days

$n$

10

16

Mean (SD)

$1784(1851)$

$1503(1604)$

VKA dose, mg

Mean (SD)

$4.9(2)$

$4.1(2.1)$

Indication for VKA treatment, $n(\%)$

Atrial fibrillation

$11(55)$

$16(72)$

Cerebral ischemia

$3(15)$

$1(4.5)$

Cardiac failure congestive

$1(5)$

0

Pulmonary embolism

$2(9.1)$

Aortic valve replacement

$1(5)$

$1(4.5)$

Deep vein thrombosis

$1(5)$

$2(9.1)$

Sick sinus syndrome

$1(5)$

$1(5)$

0

Coronary artery disease

$1(5)$

0

Site of GI bleed ${ }^{\mathrm{a}}, n$ (\%)

$\begin{array}{lcc}\text { Upper GI } & 3(10) & 4(18.2) \\ \text { Lower GI } & 3(15) & 5(22.7) \\ \text { Unspecified/data unavailable } & 14(75) & 13(59.1)\end{array}$

${ }^{a}$ Where bleeding site was given as "probably upper," site of bleed was considered unspecified. 4F-PCC, 4-factor prothrombin complex concentrate; GI, gastrointestinal; Hct, hematocrit; Hgb, hemoglobin; INR, international normalized ratio; SD, standard deviation; SFH, Seton Family of Hospitals, Austin, Texas; VKA, vitamin K antagonist; URMC, University of Rochester Medical Center, Rochester, New York.

Median (IQR) times were $21.1(16.4,28.8) \mathrm{h}$ for $4 \mathrm{~F}-\mathrm{PCC}$ and $29.7(23.2,66.3) \mathrm{h}$ for plasma.

The mean time between start of study treatment and first procedure was also significantly shorter for the 4F-PCC group than the plasma group $(P=0.037)$, as shown in 


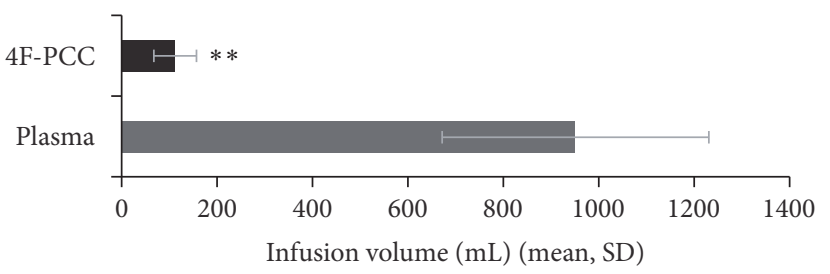

(a)

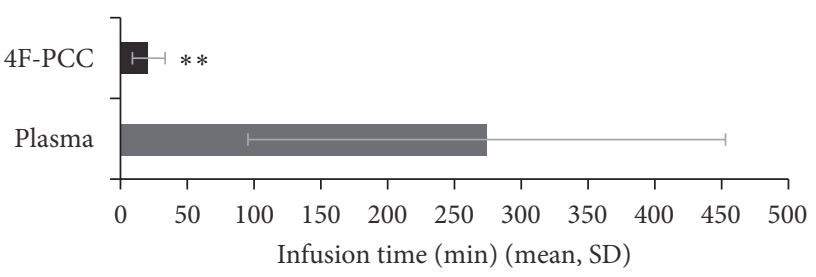

(b)

Figure 1: (a) Infusion volumes of study treatments. (b) Infusion times of study treatments. ${ }^{* *} P<0.0001$ compared with plasma. 4F-PCC, 4-factor prothrombin complex concentrate; min, minute; SD, standard deviation.

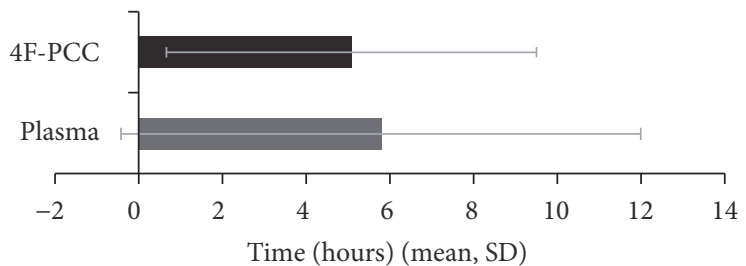

(a)

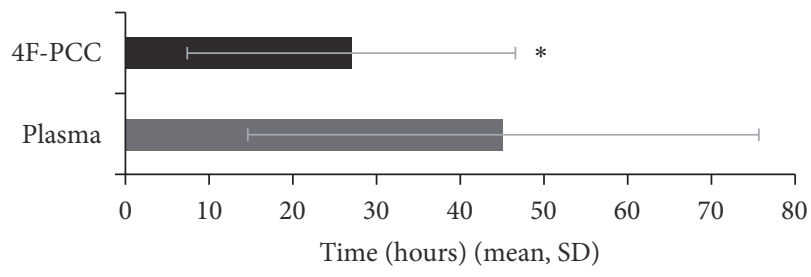

(b)

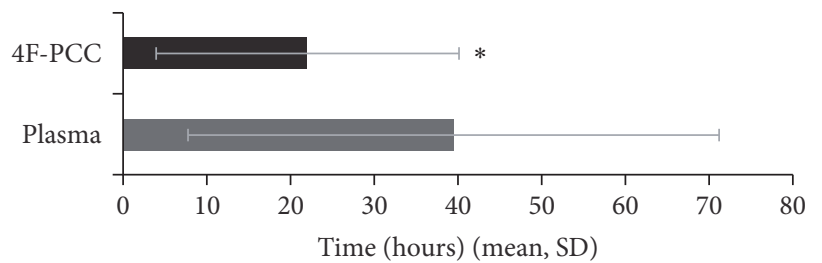

(c)

Figure 2: (a) Time between admission and start of study treatment. (b) Time between admission and first procedure. (c) Time between start of study treatment and first procedure. $P$ value for comparison of groups for times from admission to study treatment $=0.64 .{ }^{*} P<0.05$ compared with plasma. 4F-PCC, 4 -factor prothrombin complex concentrate; SD, standard deviation.

TABLE 3: Study treatment doses.

\begin{tabular}{lc}
\hline & Patients, $n(\%)$ \\
\hline Plasma dose $(n=20)$ & \\
$10 \mathrm{~mL} / \mathrm{kg}$ & $11(55)$ \\
$12 \mathrm{~mL} / \mathrm{kg}$ & $4(20)$ \\
$15 \mathrm{~mL} / \mathrm{kg}$ & $5(25)$ \\
$4 \mathrm{~F}-\mathrm{PCC}$ dose $(n=22)$ & \\
$25 \mathrm{IU} / \mathrm{kg}$ & $11(50)$ \\
$35 \mathrm{IU} / \mathrm{kg}$ & $4(18)$ \\
$50 \mathrm{IU} / \mathrm{kg}$ & $7(32)$ \\
\hline 4 F-PCC, 4-factor prothrombin complex concentrate.
\end{tabular}

Figure 2(c). Median (IQR) times were $17.5(12.8,22.8) \mathrm{h}$ for 4F-PCC and $23.9(18.5,62.0) \mathrm{h}$ for plasma.

The median (IQR) total hospital stay duration was not different between treatment groups $(6.0[4.0,10.5]$ and 5.0 $[4.0,8.0]$ days for 4F-PCC and plasma, resp.; $P=0.69)$. Mean (standard deviation) hospital stay duration (based on available data) was 8.1 (6.2) days in the 4F-PCC group $(n=20)$ and $7.7(7.2)$ days in the plasma group $(n=19)$.
3.4. Surgical/Invasive Procedures. In this post hoc analysis, the urgent interventional procedures that patients underwent were all endoscopic procedures and included EGD, colonoscopy, and sigmoidoscopy. Full details regarding the types of procedures that patients underwent are provided in Supplementary Material available online at https://doi.org/10.1155/2017/8024356.

3.5. Nonstudy Blood Product Use. The numbers of patients who required nonstudy blood products, including packed red blood cells (PRBCs), and nonstudy plasma were comparable between the two groups. In the 4F-PCC group, $68 \%$ of patients $(15 / 22)$ required $\mathrm{PRBC}$ transfusion, compared with $60 \%(12 / 20)$ in the plasma group. Two patients in the 4F-PCC group (9\%) required nonstudy plasma compared with 3 (15\%) from the plasma group.

3.6. Efficacy. Results for the primary and coprimary study endpoints of effective hemostasis and rapid INR reduction are described in Table 4 . In the GI bleeds cohort, as in the original studies $[16,17]$, rapid INR reduction $(\leq 1.3$ at $0.5 \mathrm{~h}$ after infusion end) was observed more frequently in the 4F-PCC group than the plasma group $(P<0.0001)$. 
TABLE 4: Efficacy endpoints from the original phase IIIb studies.

\begin{tabular}{|c|c|c|c|}
\hline & $\begin{array}{l}4 \mathrm{~F}-\mathrm{PCC} \\
(n=22)\end{array}$ & $\begin{array}{l}\text { Plasma } \\
(n=20)\end{array}$ & $P$ value \\
\hline \multicolumn{4}{|l|}{ Primary endpoint } \\
\hline \multicolumn{4}{|l|}{ Hemostatic efficacy $^{\mathrm{a}}, n(\%)$} \\
\hline Excellent & $12(55)$ & $11(55)$ & \\
\hline Good & $4(18)$ & $4(20)$ & 0.98 \\
\hline Poor/none & $6(27)$ & $5(2.05)$ & \\
\hline $\begin{array}{l}\text { Coprimary endpoint } \\
\text { Rapid INR reduction }^{\mathrm{b}}, n(\%)\end{array}$ & $13(65)$ & 0 & $<0.0001$ \\
\hline $\begin{array}{l}\text { Secondary endpoint: time } \\
\text { from start of infusion to INR } \\
\text { reduction } \leq 1.3 \text {, hours, mean } \\
\text { (SD) }\end{array}$ & $2.6(5.5)$ & $14.3(8.8)$ & 0.0001 \\
\hline
\end{tabular}

${ }^{a}$ In both studies, hemostatic efficacy was assessed by an independent Endpoint Adjudication Board: over a 24-hour period from the start of infusion (bleeding study) or from the start of infusion to the end of procedure (surgery study), as excellent, good, or poor/none. (Surgery study hemostatic efficacy was originally reported as a binary endpoint [effective or noneffective] as assessed from the start of infusion to the end of the procedure, and effective hemostasis was defined as intraoperative blood loss not exceeding predicted blood loss by $30 \%$ or $50 \mathrm{~mL}$, normal or mildly abnormal hemostasis [surgeon assessed], and no administration of nonstudy coagulation products). $\mathrm{b}^{\mathrm{b}} \leq 1.3$ at $30 \mathrm{~min}$ after end of infusion. 4F-PCC, 4 -factor prothrombin complex concentrate; INR, international normalized ratio; min, minutes; SD, standard deviation.

Furthermore, patients in the 4F-PCC group achieved INR $\leq 1.3$ more rapidly after the start of infusion than those in the plasma group $(P=0.0001)$. Unlike the original studies, in the small GI bleeds cohort, there was no difference in hemostatic efficacy between groups.

3.7. Safety. Safety outcomes were consistent with the results from the two original multicenter clinical trials $[16,17,23]$. Adverse events (AEs) were reported for $15 / 22$ patients (68\%) in the $4 \mathrm{~F}-\mathrm{PCC}$ group and $17 / 20$ patients $(85 \%)$ in the plasma group. Serious AEs were reported for $4 / 20$ patients (18\%) in the $4 \mathrm{~F}$-PCC group and $7 / 22$ patients (35\%) in the plasma group. There was 1 fluid overload event in the 4F-PCC group (5\%) and 4 in the plasma group (20\%); no events in either group were deemed related to study treatment by the investigator. Thromboembolic events were experienced by 1 patient in the 4F-PCC group (5\%) and 2 patients $(10 \%)$ in the plasma group; one of the events in the plasma group was considered treatment-related. There were no deaths in either group. Safety results are tabulated in Supplementary Material (Additional File 2: Table S2).

\section{Discussion}

We found that time between start of infusion and start of procedure was significantly shorter in patients who received 4 F-PCC compared with those in the plasma group. This reduced time to procedure was likely a consequence of the more rapid INR reduction and smaller infusion volume and therefore shorter infusion time with $4 \mathrm{~F}-\mathrm{PCC}$ versus plasma. The GI bleeds patient cohort mirrored the parent studies in that more of the 4F-PCC group than the plasma group met the coprimary endpoint of rapid INR reduction, demonstrating the efficacy of 4F-PCC. Though we did not see a similar effect on hemostatic efficacy, patient numbers in this study, as noted, were small.

It is important to establish whether a reduction in time to procedure is due to association or causation. Other factors which may affect time to procedure include hospital protocols guiding the initial management of patients with GI bleeding, the hemodynamic stability of the patient, and resource availability, including whether a 24-hour endoscopy service is available. However, as this analysis was performed using data from only 2 sites, variability in everything but individual patients should be limited.

Though in this small cohort we did not observe a significant difference in time between admission and start of study treatment between 4F-PCC- and plasma-treated patient groups, we speculate that a study of a larger sample would find a shorter time to treatment with 4F-PCC. This speculation is based on the established delays associated with plasma use, which requires blood typing and thawing [9], and partly due to sample size considerations. We note that our study reflects clinical trial practice rather than routine clinical practice, and as such all patients would have experienced minor delays associated with trial participation caused by review of inclusion/exclusion criteria, consent, and randomization to treatment. Therefore, in routine clinical practice, time to treatment with either plasma or $4 \mathrm{~F}-\mathrm{PCC}$ would likely be shorter than that found in this study. Though availability of thawed plasma in an emergency department reduces time to plasma infusion [24], we suggest that the larger infusion volume/longer infusion time with plasma versus $4 \mathrm{~F}-\mathrm{PCC}$ and the shorter time to reverse INR with 4F-PCC versus plasma will nonetheless consistently lead to an overall longer time to procedure with plasma versus 4F-PCC treatment in routine clinical practice.

4.1. Reducing Time to Procedure Could Improve Patient Outcomes. While the frequency of serious AEs and fluid overload was too small for meaningful statistical testing, our results appear consistent with the overall results of both trials. Plasma use is associated with an increased risk of fluid overload $[9,25]$. This has been associated with increased hospital costs and increased length of hospital stay $[25,26]$. In the setting of a study with a larger number of patients than the present analysis, this safety consideration could potentially contribute to reduced mean hospital stay in patients treated with 4F-PCC versus plasma. No relationship between frequency of fluid overload events and dose was noted for either plasma or 4F-PCC in the total study cohorts [23], and the low number of events $(n=5)$ in the population of patients with GI bleeds included here precludes any further specific analysis. Though there were no deaths in this cohort, mortality due to GI bleeding could potentially be reduced by expediting diagnosis and bleeding control with earlier diagnostic and therapeutic endoscopy. This is supported by a prospective analysis of patients with GI bleeding, which identified the lone independent risk factor associated with 
TABLE 5: Analyses of benefits associated with early intervention in patients with GI bleeding.

\begin{tabular}{|c|c|c|c|c|}
\hline \multirow[b]{2}{*}{ Study } & \multirow[b]{2}{*}{ Study design } & \multicolumn{3}{|c|}{ Outcome with early intervention } \\
\hline & & $\begin{array}{l}\text { Cost benefit/improved } \\
\text { health resource } \\
\text { utilization }\end{array}$ & $\begin{array}{c}\text { Reduced } \\
\text { hospital LOS }\end{array}$ & $\begin{array}{c}\text { Improved } \\
\text { clinical } \\
\text { outcomes }\end{array}$ \\
\hline \multirow{4}{*}{$\begin{array}{l}\text { Lee et al., } \\
1999[20]\end{array}$} & (i) Prospective RCT & \multirow{4}{*}{ Yes } & \multirow{4}{*}{ Yes } & \multirow{4}{*}{ No } \\
\hline & $\begin{array}{l}\text { (ii) Patients admitted with nonvariceal upper GI bleeding were } \\
\text { randomized to either }\end{array}$ & & & \\
\hline & (a) control group (elective endoscopy within $1-2$ days; $n=54$ ) & & & \\
\hline & (b) early endoscopy group (endoscopy within $1-2 \mathrm{~h} ; n=56$ ) & & & \\
\hline \multirow{4}{*}{$\begin{array}{l}\text { Bjorkman et } \\
\text { al., } 2004[21]\end{array}$} & (i) Prospective, randomized, blinded, multicenter trial & \multirow{4}{*}{ No } & \multirow{4}{*}{ No } & \multirow{4}{*}{ No } \\
\hline & $\begin{array}{l}\text { (ii) Patients with nonvariceal upper GI bleeding were randomized } \\
\text { to either }\end{array}$ & & & \\
\hline & (a) elective endoscopy (within $48 \mathrm{~h}$ of initial evaluation; $n=46$ ) & & & \\
\hline & (b) urgent endoscopy (within $6 \mathrm{~h}$ of initial evaluation; $n=47$ ) & & & \\
\hline \multirow{3}{*}{$\begin{array}{l}\text { Lim et al., } \\
2011[22]\end{array}$} & $\begin{array}{l}\text { (i) Prospective single-center trial enrolled } 934 \text { patients with } \\
\text { nonvariceal upper GI bleeding }\end{array}$ & \multirow{3}{*}{ N/A } & \multirow{3}{*}{ N/A } & \multirow{3}{*}{$\begin{array}{l}\text { Yes (reduced } \\
\text { mortality in } \\
\text { high-risk } \\
\text { patients) }\end{array}$} \\
\hline & $\begin{array}{l}\text { (ii) Lone independent risk factor associated with all-cause mortality } \\
\text { in high risk patients }(G B S \geq 12 \text { ) was found to be the time lapse } \\
\text { between presentation and endoscopy }\end{array}$ & & & \\
\hline & (iii) This association was not replicated in low-risk patients $(\mathrm{GBS}<12)$ & & & \\
\hline
\end{tabular}

all-cause mortality in high risk patients (Glasgow-Blatchford Score $\geq 12$ ) to be the time lapse between presentation and endoscopy [22] (Table 5).

4.2. Resource Use in Patients with GI Bleeds. GI bleeding may require hospitalization, laboratory tests, blood product use, diagnostic workup, and interventional procedures, potentially including surgery. A systematic review of patients with upper and lower GI bleeding demonstrated substantial healthcare costs associated with these conditions [27]. In this analysis, fewer patients received $\mathrm{PRBC}$ transfusions in the plasma group than the 4F-PCC group; however, given our small sample size, conclusive evidence for this should come from larger, future investigations.

In patients with GI bleeding, cost benefits may be expected from reducing hospital length of stay and the need for additional treatments or procedures (such as blood transfusions and further endoscopic or surgical intervention). However, the available data regarding cost benefits associated with early intervention in GI bleeding are conflicting [20-22] (Table 5). In the present analysis, reduced time to procedure was not associated with shorter hospital stay. Potential explanations for this include small sample size, comorbidities, and social factors affecting discharge.

4.3. 4F-PCC Impact on Procedural Efficacy in Patients with GI Bleeds. Given the improvement in time to procedure with 4F-PCC, analyses of association between time to surgical or invasive procedures and the efficacy of such procedures (assessed via incidence of rebleeding) would be of interest. Though this was beyond the scope of our investigation, it is something that should be addressed by future work.
4.4. Strengths and Limitations. The key limitation of this study is, as mentioned, its small sample size, as only a subset of patients from two larger studies were included. As this was a post hoc analysis, results cannot be considered conclusive and should be viewed as hypothesis-generating. In this study we benefitted from being able to draw on data not just for 4F-PCC-treated patients but also for a plasmatreated control group, to provide a balanced analysis of the outcomes tested herein.

\section{Conclusions}

Overall, in this post hoc analysis in patients experiencing GI bleeding while being treated with VKA anticoagulants, leading to a requirement for urgent surgical or invasive procedures, 4F-PCC was associated with smaller infusion volumes, shorter infusion times, and shorter times from admission to procedure, compared with plasma.

\section{Ethical Approval}

Both studies were approved by the independent ethics committees (IECs) and institutional review boards (IRBs) of the participating centers and conducted in accordance with local ethics regulations. For University of Rochester Medical Center, the IEC/IRB was the Western Institutional Review Board and, for the Seton Family of Hospitals, the IEC/IRB was the Austin Multi-IRB.

\section{Consent}

Written informed consent was obtained from or on behalf of all patients. 


\section{Conflicts of Interest}

Majed A. Refaai has received consulting fees from CSL Behring and is a member of a CSL Behring speakers' bureau. Ravi Sarode has received consulting fees and honoraria from CSL Behring and has served as a member of advisory boards for Alexion, Instrument Laboratories, Kedrion, and Octapharma. Joshua N. Goldstein has received consulting fees and a research grant from CSL Behring and has served on an advisory board for Bristol-Myers Squibb. Andres Brainsky and Laurel Omert are employees of CSL Behring, and Martin L. Lee and Truman J. Milling have received consulting fees from CSL Behring. Truptesh H. Kothari, Shana Straub, and Jacob Falcon have no conflicts of interest or disclosures to report.

\section{Acknowledgments}

The studies were sponsored by CSL Behring. Medical writing assistance was provided by Margarita Lens of Fishawack Communications Ltd. and funded by the sponsor.

\section{References}

[1] C. P. Choudari and K. R. Palmer, "Acute gastrointestinal haemorrhage in patients treated with anticoagulant drugs," Gut, vol. 36, no. 4, pp. 483-484, 1995.

[2] R. Sarode, K. Matevosyan, R. Bhagat, C. Rutherford, C. Madden, and J. E. Beshay, "Rapid warfarin reversal: A 3-factor prothrombin complex concentrate and recombinant factor VIIa cocktail for intracerebral hemorrhage: Clinical article," Journal of Neurosurgery, vol. 116, no. 3, pp. 491-497, 2012.

[3] S. Schulman, R. J. Beyth, C. Kearon, and M. N. Levine, "Hemorrhagic complications of anticoagulant and thrombolytic treatment: American College of Chest Physicians evidence-based clinical practice guidelines (8th edition)," Chest, vol. 133, no. 6, pp. 257S-298S, 2008.

[4] N. Shehab, L. S. Sperling, S. R. Kegler, and D. S. Budnitz, "National estimates of emergency department visits for hemorrhage-related adverse events from clopidogrel plus aspirin and from warfarin," Archives of Internal Medicine, vol. 170, no. 21, pp. 1926-1933, 2010.

[5] C. S. Landefeld and R. J. Beyth, "Anticoagulant-related bleeding: Clinical epidemiology, prediction, and prevention," The American Journal of Medicine, vol. 95, no. 3, pp. 315-328, 1993.

[6] G. Palareti, N. Leali, S. Coccheri et al., "Bleeding complications of oral anticoagulant treatment: An inception-cohort, prospective collaborative study (ISCOAT)," Lancet, vol. 348, no. 9025, pp. 423-428, 1996

[7] B. A. Steinberg, D. N. Simon, L. Thomas et al., "Management of Major Bleeding in Patients With Atrial Fibrillation Treated With Non-Vitamin K Antagonist Oral Anticoagulants Compared With Warfarin in Clinical Practice (from Phase II of the Outcomes Registry for Better Informed Treatment of Atrial Fibrillation [ORBIT-AF II])," American Journal of Cardiology, vol. 119, no. 10, pp. 1590-1595, 2017.

[8] L. Yang, S. Stanworth, S. Hopewell, C. Doree, and M. Murphy, "Is fresh-frozen plasma clinically effective? An update of a systematic review of randomized controlled trials (CME)," Transfusion, vol. 52, no. 8, pp. 1673-1686, 2012.
[9] W. Ageno, A. S. Gallus, A. Wittkowsky, M. Crowther, E. M. Hylek, and G. Palareti, "Oral anticoagulant therapy: antithrombotic therapy and prevention of thrombosis, 9th ed: American College of Chest Physicians evidence-based clinical practice guidelines," Chest, vol. 141, no. 2, pp. e44S-e88S, 2012.

[10] R. Sarode, L. Holland, T. E. Warkentin, M. Refaai, M. A. Crowther, and M. A. Johnston, "Suboptimal effect of a threefactor prothrombin complex concentrate (Profilnine-SD) in correcting supratherapeutic international normalized ratio due to warfarin overdose," Transfusion, vol. 49, no. 6, pp. 1171-1177, 2009.

[11] Baxter, "BEBULIN (Factor IX Complex), Nanofiltered and Vapor Heated," 2012, https://www.baxalta.com/assets/documents/bebulin_pi.pdf.

[12] CSL Behring, "Kcentra ${ }^{\circledR}$ Highlights of Prescribing Information," 2013, http://labeling.cslbehring.com/PI/US/Kcentra/EN/Kcentra-Prescribing-Information.pdf.

[13] Grifols Biologicals Inc, "Factor IX Complex, Profilnine SD Prescribing Information,” 2010, http:/www.fda.gov/ucm/groups/ fdagov-public/@fdagov-bio-gen/documents/document/ucm261964.pdf.

[14] Octapharma, "Octaplex Summary of Product Characteristics," 2013, https://www.medicines.org.uk/emc/medicine/21897.

[15] CSL Behring, "Beriplex P/N Summary of Product Characteristics," 2016, https://www.medicines.org.uk/emc/medicine/20797.

[16] J. N. Goldstein, M. A. Refaai, T. J. Milling Jr. et al., "Fourfactor prothrombin complex concentrate versus plasma for rapid vitamin $\mathrm{K}$ antagonist reversal in patients needing urgent surgical or invasive interventions: a phase $3 \mathrm{~b}$, open-label, noninferiority, randomised trial," The Lancet, vol. 385, no. 9982, pp. 2077-2087, 2015.

[17] R. Sarode, T. J. Milling, M. A. Refaai et al., "Efficacy and safety of a 4-factor prothrombin complex concentrate in patients on vitamin $\mathrm{K}$ antagonists presenting with major bleeding: a randomized, plasma-controlled, phase iiib study," Circulation, vol. 128, no. 11, pp. 1234-1243, 2013.

[18] I. Pabinger, A. Tiede, U. Kalina, S. Knaub, R. Germann, and H. Ostermann, "Impact of infusion speed on the safety and effectivenes of prothrombin complex concentrate A prospective clinical trial of emergency anticoagulation reversal," Annals of Hematology, vol. 89, no. 3, pp. 309-316, 2010.

[19] J. Ansell, J. Hirsh, E. Hylek, A. Jacobson, M. Crowther, and G. Palareti, "Pharmacology and management of the vitamin K antagonists: American College of Chest Physicians EvidenceBased Clinical Practice Guidelines (8th Edition)," Chest, vol. 133, no. 6, pp. 160S-198S, 2008.

[20] J. G. Lee, S. Turnipseed, P. S. Romano et al., "Endoscopy-based triage significantly reduces hospitalization rates and costs of treating upper GI bleeding: A randomized controlled trial," Gastrointestinal Endoscopy, vol. 50, no. 6, pp. 755-761, 1999.

[21] D. J. Bjorkman, A. Zaman, M. B. Fennerty, D. Lieberman, J. A. DiSario, and G. Guest-Warnick, "Urgent vs. elective endoscopy for acute non-variceal upper-GI bleeding: an effectiveness study," Gastrointestinal Endoscopy, vol. 60, no. 1, pp. 1-8, 2004.

[22] L. G. Lim, K. Y. Ho, Y. H. Chan et al., "Urgent endoscopy is associated with lower mortality in high-risk but not low-risk nonvariceal upper gastrointestinal bleeding," Endoscopy, vol. 43, no. 4, pp. 300-306, 2011.

[23] T. J. Milling Jr., M. A. Refaai, and J. N. Goldstein, “Thromboembolic events after vitamin $\mathrm{K}$ antagonist reversal with 4 -factor prothrombin complex concentrate: exploratory analyses of two 
randomized, plasma-controlled studies," Annals of Emergency Medicine, vol. 67, no. 1, pp. 96-105.e5, 2016.

[24] Z. A. Radwan, Y. Bai, N. Matijevic et al., "An emergency department thawed plasma protocol for severely injured patients," JAMA Surgery, vol. 148, no. 2, pp. 170-175, 2013.

[25] G. Magee and A. Zbrozek, "Fluid overload is associated with increases in length of stay and hospital costs: Pooled analysis of data from more than 600 US hospitals," ClinicoEconomics and Outcomes Research, vol. 5, no. 1, pp. 289-296, 2013.

[26] G. Magee, C. Peters, and A. Zbrozek, "Analysis of inpatient use of fresh frozen plasma and other therapies and associated outcomes in patients with major bleeds from vitamin $\mathrm{K}$ antagonism," Clinical Therapeutics, vol. 35, no. 9, pp. 1432-1443, 2013.

[27] D. R. Parker, X. Luo, J. J. Jalbert, and A. R. Assaf, "Impact of upper and lower gastrointestinal blood loss on healthcare utilization and costs: a systematic review," Journal of Medical Economics, vol. 14, no. 3, pp. 279-287, 2011. 


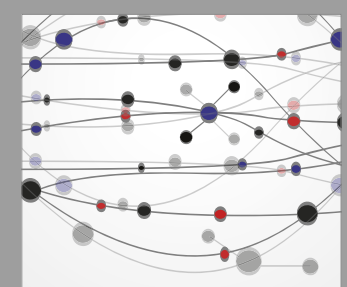

The Scientific World Journal
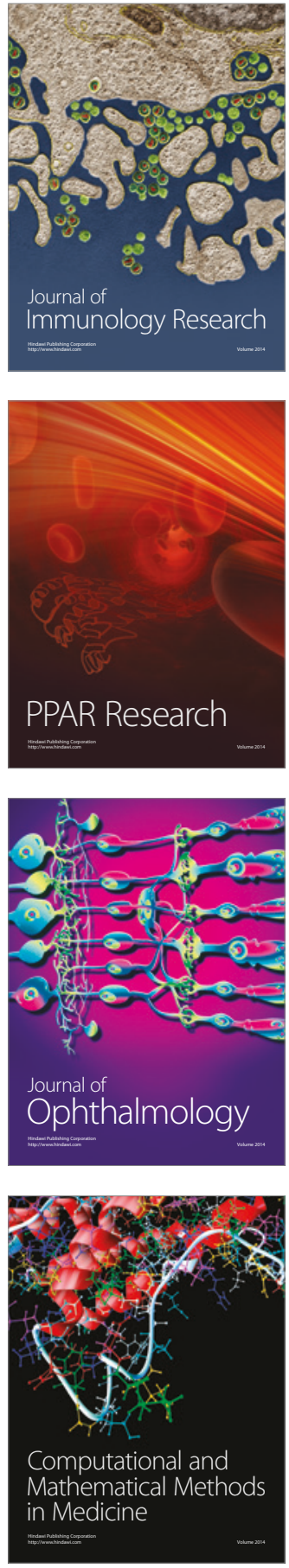

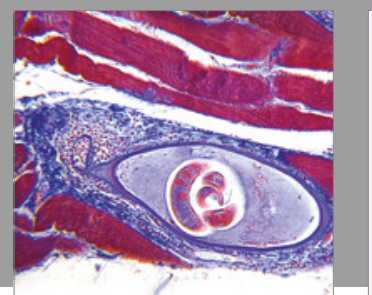

Gastroenterology Research and Practice
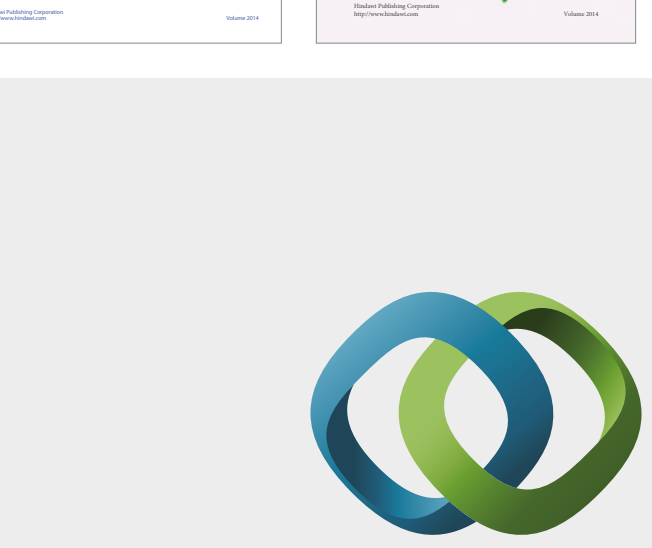

\section{Hindawi}

Submit your manuscripts at

https://www.hindawi.com
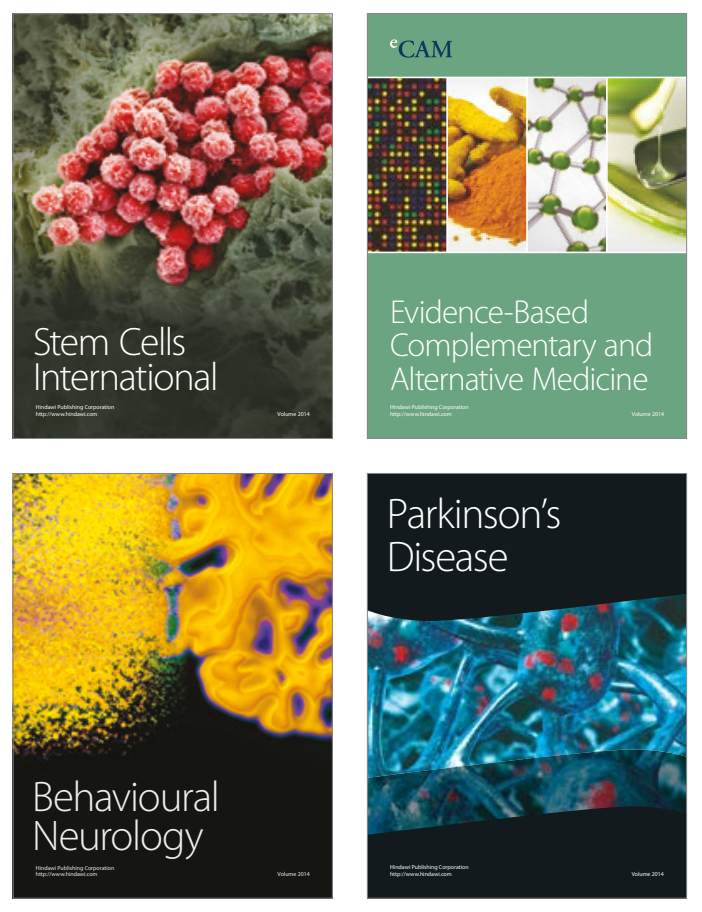
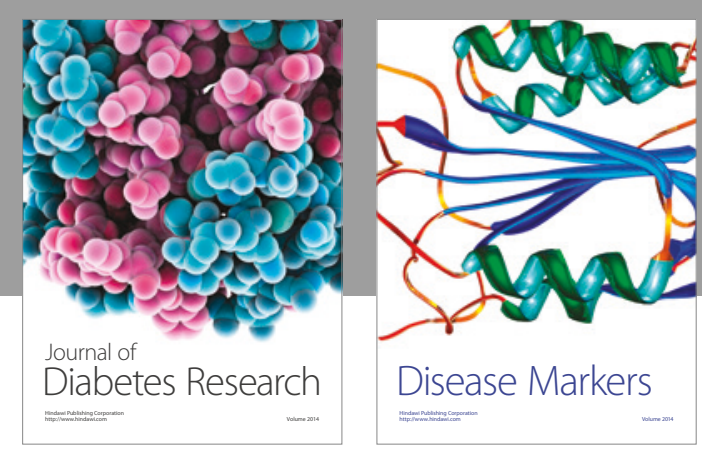

Disease Markers
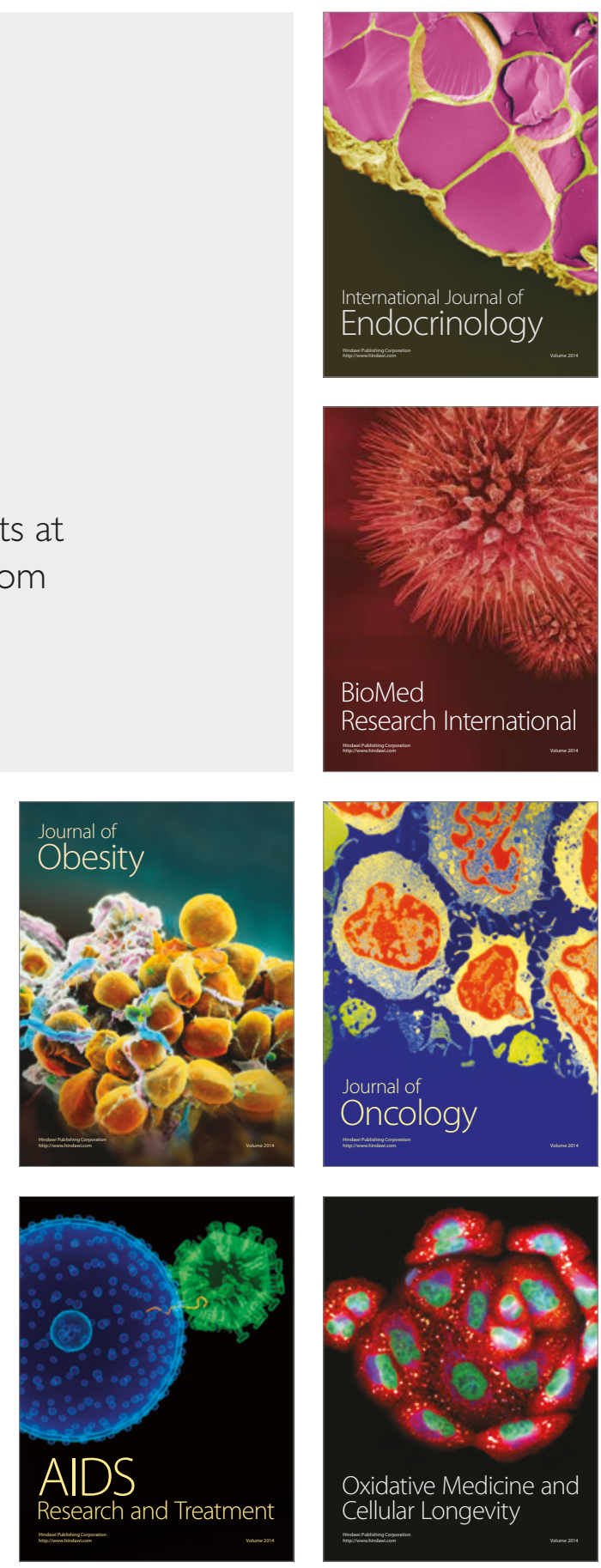